\title{
Characterization of Kurtz randomness by a differentiation theorem
}

\author{
Kenshi Miyabe
}

July 23, 2012

\begin{abstract}
Brattka, Miller and Nies [5] showed that some major algorithmic randomness notions are characterized via differentiability. The main goal of this paper is to characterize Kurtz randomness by a differentiation theorem on a computable metric space. The proof shows that integral tests play an essential part and shows that how randomness and differentiation are connected.
\end{abstract}

\section{Introduction}

This work is a continuation of Brattka, Miller and Nies [5], which showed that some major algorithmic randomness notions are characterized via differentiability.

\section{$1.1 \quad$ Differentiation of integrals}

Lebesgue [15] showed that, if $f$ is integrable on the real line, the derivative of $F(x)=\int_{-\infty}^{x} f(t) d t$ exists and is equal to $f(x)$ almost everywhere. The points for which this equality holds are called Lebesgue points. This theorem was generalized to the Lebesgue measure on $\mathbb{R}^{n}$ in Lebesgue [16] and the generalized theorem is called Lebesgue Differentiation Theorem. The theorem was further generalized to any locally finite Borel measure on $\mathbb{R}^{n}$ by Besicovitch [1] and this theorem is called the differentiation theorem.

\subsection{Characterization via differentiability}

Algorithmic randomness defines random points on the unit interval as the points that avoid some kind of effectively null sets. For example, Martin-Löf randomness is defined as follows. A Martin-Löf test is a sequence $\left\{U_{n}\right\}$ of uniformly c.e. open sets with $\mu\left(U_{n}\right) \leq 2^{-n}$ where $\mu$ is a computable measure on the space such as Lebesgue measure on the unit interval. A point $x$ is Martin-Löf random if it passes the all tests, that is, $x \notin \bigcap_{n} U_{n}$. Other randomness notions 
are Schnorr randomness, computable randomness, Kurtz randomness and weak 2-randomness. See [7] and [18] for details.

It is natural to ask whether a function in a class is always differentiable at an algorithmic random point. Demuth [6] showed that a real is Martin-Löf random if and only if every computable function of bounded variation is differentiable at the point. Then "only if" direction is an effective form of Lebesgue's theorem. Furthermore, Brattka, Miller and Nies [5] gave characterizations via differentiability of computable randomness, weak 2-randomness and Martin-Löf randomness (recast). A version of Schnorr randomness was given by Pathak, Rojas and Simpson [19] and independently by Jason Rute. Another characterization of Schnorr randomness via differentiability was showed by Freer, Kjos-Hanssen and Nies [8].

Note that differentiability is weaker than the differentiation theorem. Differentiability requires only the existence of the limit but the differentiation theorem says that the limit exists and is equal to the value of the original function. Furthermore, the differentiation theorem has potential to be generalized to more general spaces. A goal of this paper is to give a characterization of Kurtz randomness by a differentiation theorem. In the proof integral tests play an essential part.

We need to give a remark here. The infinitely dimensional version of the differentiation theorem does not hold in general: there is a Gaussian measure $\mu$ together with an integrable function $f$ on a separable Hilbert space $H$ such that

$$
\lim _{s \rightarrow 0} \inf \left\{\frac{1}{\mu B(x, r)} \int_{B(x, r)} f d \mu: x \in H, 0<r<s\right\}=+\infty .
$$

See Tiser [21] for the detail. This means that the differentiation theorem on a metric space with a Borel measure does not hold in general. So if one expects some positive results on a general space, one needs some restriction. One sufficient condition is continuity because, if a function is continuous, then all points are Lebesgue points for the function. Thus, all points are Lebesgue points for a computable function. We will propose a little larger class of almost everywhere computable functions so that the set of Lebesgue points for every function in the class is equivalent to the set of Kurtz random points.

\subsection{Randomness on a computable metric space}

Algorithmic randomness is usually studied on the Cantor space or the unit interval. Computable analysis [23, 24] generalized computability to a more general space. Algorithmic randomness on a computable metric space was also studied in some literature $[9,13,11,12,10]$. The studied randomness notions are usually Martin-Löf Randomness or Schnorr randomness while Hoyrup and Rojas [13, Lemma 6.2.1] essentially showed that Martin-Löf random points are contained in Kurtz random points on a computable metric space. This result also follows from our characterization of Kurtz randomness. 


\subsection{Overview of this paper}

In Section 2 we recall some results from computable analysis. In Section 3 we give some characterizations by integral tests of Kurtz randomness and give a characterization by a differentiation theorem. In Section 4 we introduce almost everywhere computability and remove non-negativeness and extendedness in the characterization. In Section 5 we discuss when two functions are equal on Kurtz points.

\section{Preliminaries}

We recall some notions from computable analysis. See [23, 3, 4, 24] for details. We use "iff" to mean "if and only if".

\subsection{Computable analysis}

Let $\Sigma$ be a finite alphabet such that $0,1 \in \Sigma$. By $\Sigma^{*}$ we denote the set of finite words over $\Sigma$ and by $\Sigma^{\omega}$ the set of infinite sequences over $\Sigma$. A notation of a set $X$ is a surjective partial function $\nu: \subseteq \Sigma^{*} \rightarrow X$, and a representation is a surjective partial function $\delta: \subseteq \Sigma^{\omega} \rightarrow X$. A naming system is a notation or a representation. Let $Y_{1}, Y_{2} \in\left\{\Sigma^{*}, \Sigma^{\omega}\right\}$. Let $\gamma_{i} \subseteq Y_{i} \rightarrow X_{i}$ be naming systems. A point $x \in X_{1}$ is $\gamma$-computable if it has a computable $\gamma$-name. A function $h: \subseteq Y_{1} \rightarrow Y_{2}$ realizes a partial function $f: \subseteq X_{1} \rightarrow X_{2}$ iff $\gamma_{2} \circ h\left(y_{1}\right)=f \circ \gamma_{1}\left(y_{1}\right)$ whenever $y_{1} \in \operatorname{dom}\left(\gamma_{1}\right)$ and $\gamma_{1}\left(y_{1}\right) \in \operatorname{dom}(f)$. The function $f$ is $\left(\gamma_{1}, \gamma_{2}\right)$ computable iff it has a computable realization.

Definition 2.1 (computable metric space). A computable metric space is a 3-tuple $\mathbf{X}=(X, d, \alpha)$ such that

(i) $(X, d)$ is a metric space,

(ii) $\alpha: \subseteq \Sigma^{*} \rightarrow A$ is a notation of a dense subset $A$ of $X$ with a computable domain,

(iii) d restricted to $A \times A$ is $(\alpha, \alpha, \rho)$-computable.

We give some examples of computable metric spaces.

Example 2.2. (i) (unit interval) Let $\mathbf{I}=(\mathbb{I}, d, \alpha)$ be such that $\mathbb{I}=[0,1], \alpha$ is a canonical notation of $\mathbb{Q} \cap \mathbb{I}$ and $d(p, q)=|p-q|$.

(ii) (real line) Let $\mathbf{R}=(\mathbb{R}, d, \alpha)$ be such that $\alpha$ is a canonical notation of $\mathbb{Q}$ and $d(p, q)=|p-q|$.

(iii) (extended real line) $\overline{\mathbf{R}}=(\overline{\mathbb{R}}, d, \alpha)$ be such that $\overline{\mathbb{R}}=\mathbb{R} \cup\{ \pm \infty\}$, $\alpha$ is a canonical notation of $\mathbb{Q} \cup\{ \pm \infty\}$ and $d(x, y)=|f(x)-f(y)|$ where $f(x)=\frac{x}{1+|x|}, f(\infty)=1$ and $f(-\infty)=-1$. 
The canonical notations of the natural and the rational numbers are denoted by $\nu_{\mathbb{N}}$ and $\nu_{\mathbb{Q}}$, respectively. The representation $\rho_{<}: \subseteq \Sigma^{\omega} \rightarrow \mathbb{R}$ is defined by

$$
\rho_{<}(p)=x \Longleftrightarrow p \text { enumerates all } q \in \mathbb{Q} \text { with } q<x .
$$

We use $\bar{\rho}_{<}$for the representation of points in $\mathbb{R} \cup\{\infty\}$. The representation $\rho: \subseteq \Sigma^{\omega} \rightarrow \mathbb{R}$ is defined by

$\rho(p)=x \Longleftrightarrow p$ encodes a sequence $\left\{q_{n}\right\}$ of rationals such that $\left|x-q_{n}\right| \leq 2^{-n}$.

A fast Cauchy sequence on a metric space is a sequence $\left\{x_{n}\right\}$ of points in the space such that $d\left(x_{n}, x_{n-1}\right) \leq 2^{-n}$. The representation $\delta: \subseteq \Sigma^{\omega} \rightarrow X$ of points in a computable metric space is defined by

$\delta(p)=x \Longleftrightarrow p$ encodes a fast Cauchy sequence $\left\{x_{n}\right\}$ that converges to $x$.

A basic open ball is denoted by $B(u, r)=\{x: d(u, x)<r\}$ and a basic closed ball is denoted by $\bar{B}(u, r)=\{x: d(u, x) \leq r\}$. The representation $\theta: \subseteq \Sigma^{\omega} \rightarrow \tau$ of open sets is defined by

$$
\begin{aligned}
& \theta(p)=W \Longleftrightarrow \\
& p \text { encodes a sequence }\left\{B_{i}\right\} \text { of basic open balls such that } W=\bigcup_{i} B_{i} .
\end{aligned}
$$

For simplicity we use the following terminology. A point is computable if it is $\delta$-computable. A open set is c.e. if it is $\theta$-computable. A closed set is co-c.e. if its complement is c.e. A total function $f: X_{1} \rightarrow X_{2}$ is computable if it is $\left(\delta_{1}, \delta_{2}\right)$-computable. A total function $f: X \rightarrow \overline{\mathbb{R}}$ is lower semi-computable if it is $\left(\delta, \bar{\rho}_{<}\right)$-computable. A total function $f: X \rightarrow \overline{\mathbb{R}}$ is extended computable if it is $(\delta, \bar{\rho})$-computable where $\bar{\rho}$ is the representation $\delta$ of points in $\overline{\mathbf{R}}$.

By Definition 28 and Theorem 29 in Weihrauch and Grubba [24], we have the following characterization of a computable function.

Proposition 2.3. Let $\mathbf{X}_{i}=\left(X_{i}, \tau_{i}, \beta_{i}, \nu_{i}\right)$ be computable topological spaces for $i=1,2$. For a total function $f: X_{1} \rightarrow X_{2}$, the following are equivalent:

(i) $f$ is $\left(\delta_{1}, \delta_{2}\right)$-computable,

(ii) $f^{-1}: \tau_{2} \rightarrow \tau_{1}$ is $\left(\theta_{2}, \theta_{1}\right)$-computable,

(iii) $f^{-1}\left(\nu_{2}(u)\right)$ is $\theta_{1}$-computable uniformly in $u$.

\subsection{Computable measures}

For computability of measures on a computable metric space, see [20, 2, 13]. For simplicity we only consider a probabilistic computable measure. In this paper we use the following as the definition of a computable measure.

Definition 2.4 (computable measure). A probabilistic measure $\mu$ on a computable metric space is computable if $\left.\mu\right|_{\tau}: \tau \rightarrow \mathbb{I}$ is $\left(\theta, \rho_{<}\right)$-computable (or lower semi-computable). 
See $\mathcal{M}_{C}$ in Schröder [20], $\vartheta_{\mathcal{M}}$ of Definition 2.10 in Bosserhoff [2] and Theorem 4.2.1 in Hoyrup and Rojas [13].

Proposition 2.5 ([20, Proposition 3.6], [13, Corollary 4.3.1]). Let $\mu$ be a computable measure and $f: X \rightarrow \overline{\mathbb{R}}$ be a non-negative lower semi-computable function. Then $\int f d \mu$ is lower semi-computable.

Proposition 2.6 ([13, Corollary 4.3.2]). Let $\mu$ be a computable measure, $U$ be a c.e. open set with a computable measure and $f: X \rightarrow \mathbb{R}$ be a bounded computable function. Then $\int_{U} f d \mu$ is computable.

We denote $\int_{X} f d \mu$ by $\mu(f)$.

\section{Characterization by integral tests}

In this section we give some characterizations of Kurtz randomness by integral tests and by a differentiation theorem.

Kurtz randomness or weak randomness is usually defined on Cantor space but it is easily generalized to a computable metric space with a computable measure on it.

Let $(X, d, \alpha)$ be a computable metric space and $\mu$ be a computable measure on it.

Definition 3.1 (essentially due to Kurtz [14]). A Kurtz test is a c.e. open set with measure 1. A point $x \in X$ passes a Kurtz test $U$ if $x \in U$. A point is Kurtz random if the point passes all Kurtz tests.

We use most of this section to prove the following theorem.

Theorem 3.2. For a point $z \in X$, the following are equivalent.

(i) $z$ is Kurtz random.

(ii) $f(z)<\infty$ for each non-negative extended computable function $f: X \rightarrow \overline{\mathbb{R}}$ such that $f(x)<\infty$ almost everywhere.

(iii) $f(z)<\infty$ for each non-negative extended computable function $f: X \rightarrow \overline{\mathbb{R}}$ with $\mu(f)<\infty$.

(iv) $f(z)<\infty$ for each non-negative extended computable function $f: X \rightarrow \overline{\mathbb{R}}$ such that $\mu(f)$ is a computable real.

Recall that a point $z$ is Martin-Löf random iff $f(z)<\infty$ for each nonnegative lower semicomputable function $f: X \rightarrow \overline{\mathbb{R}}$ such that $\mu(f)<\infty[22,17]$. One can see that extended computable functions are used for Kurtz randomness while lower semicomputable functions for Martin-Löf randomness.

Note that (ii) $\Rightarrow$ (iii) $\Rightarrow$ (iv) is immediate. 


\subsection{The implication (i) $\Rightarrow$ (ii)}

Proof of $(i) \Rightarrow$ (ii) of Theorem 3.2. Let $f \in \mathcal{K}$. Then the set $U_{n}=\{x \in X$ : $f(x)<n\}$ is a c.e. open set for each $n$ by 2.3. It follows that $U=\{x \in X$ : $f(x)<\infty\}=\bigcup_{n} U_{n}$ is also a c.e. open set. Since $\mu(U)=1, U$ is a Kurtz test.

If $f(x)=\infty$, then $x \notin U$ and does not pass the test. Hence $x$ is not Kurtz random.

\subsection{Some notations}

In the following we use many symbols to denote some classes of sets and functions. Most uncommon symbols are defined here or Section 4.1

Let $(X, d, \alpha)$ be a computable metric space and $\mu$ be a computable measure on it. Let $A$ be the range of $\alpha$. Then $A$ is a countable dense subset of $X$.

Let $\left\{u_{i}\right\}$ be a computable enumeration of $A$. By Lemma 2.15 in [2] or Lemma 5.11 in [13] there exists a computable sequence $\left\{r_{j}\right\}$ of reals such that $\mu\left(\bar{B}\left(u_{i}, r_{j}\right) \backslash B\left(u_{i}, r_{j}\right)\right)=0$ and $\left\{B\left(u_{i}, r_{j}\right)\right\}_{i, j}$ form a base of the topology. We fix the notion $B_{\langle i, j\rangle}$ to mean $B\left(u_{i}, r_{j}\right)$.

We call $B\left(u_{i}, r_{j}\right)$ a basic set for each $i$ and $j$. A co-basic set is the complement of a closed ball $\bar{B}^{c}\left(u_{n}, r_{n}\right)$ where $B\left(u_{n}, r_{n}\right)$ is a basic set. Note that a co-basic set is open. Let $\mathcal{I}$ be the set of all finite intersections of basic sets and co-basic sets.

Let $\mathcal{K}(U)$ be the set of non-negative extended computable functions $f: X \rightarrow$ $\overline{\mathbb{R}}$ such that $f(x)<\infty \Longleftrightarrow x \in U$. Let $\mathcal{K}$ be the set of non-negative extended computable functions $f: X \rightarrow \overline{\mathbb{R}}$ such that $f(x)<\infty$ almost everywhere. Then $f \in \mathcal{K}$ iff $f \in \mathcal{K}(U)$ for a Kurtz test $U$.

Let $\mathcal{K}_{\text {fin }}(U)$ and $\mathcal{K}_{\text {fin }}$ be the subset of $\mathcal{K}(U)$ and $\mathcal{K}$ restricted to the functions such that $\int_{U} f d \mu<\infty$ and $\mu(f)<\infty$ respectively. Similarly let $\mathcal{K}_{\text {comp }}(U)$ and $\mathcal{K}_{\text {comp }}$ be the subset of $\mathcal{K}(U)$ and $\mathcal{K}$ restricted to the functions such that $\int_{U} f d \mu$ is computable and $\mu(f)$ is computable respectively.

\subsection{Proof for the unit interval with Lebesgue measure}

In the next subsection we prove (iv) $\Rightarrow$ (i) of Theorem 3.2 for computable metric spaces. In order to make the proof more accessible, we first provide the proof for the special case of the unit interval with Lebesgue measure in this subsection.

Let $\mathbf{I}=(\mathbb{I}, d, \alpha)$ be the computable metric space of the unit interval in Example 2.2. Let $\mu$ be the Lebesgue measure on $\mathbb{I}$. Note that $\mu$ is computable.

The proof idea is as follows. From a Kurtz test $U$, we construct a function from $\mathbb{I}$ to $\overline{\mathbb{R}}$. First we divide $U$ into a pairwise disjoint sequence $\left\{U_{n}\right\}$ of uniformly c.e. open sets with $\mu\left(U_{n}\right)=2^{-n}$ by ignoring a set of rationals, that is, $U \backslash \bigcup_{n} U_{n}$ is a set of rationals. Let $V_{n}=\mathbb{I} \backslash \bigcup_{k=1}^{n} U_{k}$. Then $\mu\left(V_{n}\right)=2^{-n}$. Then $x$ passes $U \Longleftrightarrow x \notin \bigcap_{n} V_{n}$ if $x$ is not a rational. Hence the least $n$ satisfying $x \notin V_{n}$ can be called randomness deficiency of $x$ for $\left\{V_{n}\right\}$.

Roughly speaking, we construct a function $f$ by which each point approximately maps to the randomness deficiency. Then let $f_{0}$ be such that $f_{0}(x)=n$ if 
$x \in U_{n}$, and $f_{0}(x)=\infty$ otherwise. Then $f_{0}$ is non-negative and $\mu(f)=1<\infty$. However $f_{0}$ is neither continuous nor computable on some rational points and $U^{c}$.

To make the function computable at these points, we modify $f_{0}$. Recall that each open set $U_{n}$ is a union of pairwise disjoint open intervals with two rational endpoints. For each interval $(p, q)$, we construct a polygonal function $f \geq f_{0}$ with $\lim _{x \rightarrow p+} f(x)=\lim _{x \rightarrow q-} f(x)=\infty$. Intuitively, if the point $x$ is very close to $p$ or $q$, then $f(x)$ is large. Such a function $f$ will satisfy the desired property.

Before giving the proof, we prepare a lemma.

Lemma 3.3. For $p, q \in \mathbb{I} \cap \mathbb{Q}$, there exists a function $g_{\langle p, q\rangle}$ such that

(i) $g_{\langle p, q\rangle}:(p, q) \rightarrow \mathbb{R}$ is non-negative and computable uniformly in $p$ and $q$,

(ii) $\int_{(p, q)} g_{\langle p, q\rangle} d \mu$ is computable.

Proof. Let $g: \mathbb{I} \rightarrow \overline{\mathbb{R}}$ be a polygonal function satisfying the following:

(i) the set of endpoints is $\left\{1-2^{-n}: n \geq 0\right\}$,

(ii) $g\left(1-2^{-n}\right)=n$,

(iii) $g(1)=\infty$.

Note that $g$ is non-negative and extended computable. Furthermore the integration

$$
\int_{\mathbb{I}} g d \mu=\sum_{n=1}^{\infty}((n-1)+n) \cdot 2^{-n} \cdot 1 / 2=\sum_{n=1}^{\infty}(2 n-1) \cdot 2^{-n-1}
$$

exists and is computable. Let $G=\int_{\mathbb{I}} g d \mu$.

Let $g_{\langle p, q\rangle}:(p, q) \rightarrow \mathbb{R}$ be such that

$$
g_{\langle p, q\rangle}(x)= \begin{cases}g\left(\frac{(p+q) / 2-x}{(p+q) / 2-p}\right) & \text { if } x \in\left(p, \frac{p+q}{2}\right) \\ 0 & \text { if } x=\frac{p+q}{2} \\ g\left(\frac{x-(p+q) / 2}{q-(p+q) / 2}\right) & \text { if } x \in\left(\frac{p+q}{2}, q\right) .\end{cases}
$$

Then $g$ is non-negative and computable. Note that $\int_{(p, q)} g_{\langle p, q\rangle} d \mu=(q-p) G$.

Recall that $f \in \mathcal{K}_{\text {comp }}$ on the unit interval if $f: \mathbb{I} \rightarrow \overline{\mathbb{R}}$ is non-negative and extended computable such that $\mu(f)$ is computable.

In the following we often split an interval $(p, q)$ into disjoint two intervals $(p, r)$ and $(r, q)$ where $p, q, r \in \mathbb{I} \cap \mathbb{Q}$. Indeed we need not pay much attention to rationals because a rational is not Kurtz random and, for each $q \in \mathbb{I} \cap \mathbb{Q}$, there exists a function $f \in \mathcal{K}_{\text {comp }}$ such that $f(q)=\infty$. For $q=0$ or 1 , consider

$$
f(x)= \begin{cases}g_{\langle 0,1\rangle}(x) & \text { if } x \in(0,1) \\ \infty & \text { if } x=0,1\end{cases}
$$


Then $f \in \mathcal{K}_{\text {comp }}$ and $f(q)=\infty$. For $q \in(0,1)$, consider

$$
f(x)= \begin{cases}g_{\langle 0, q\rangle}(x) & \text { if } x \in(0, q) \\ g_{\langle q, 1\rangle}(x) & \text { if } x \in(q, 1) \\ \infty & \text { if } x=0, q, 1\end{cases}
$$

Then $f \in \mathcal{K}_{\text {comp }}$ and $f(q)=\infty$.

Using the function $g_{\langle p, q\rangle}$, we prove the existence of $f \in \mathcal{K}_{\text {comp }}$ for a general Kurtz test.

Proof of (iv) $\Rightarrow$ (i) of Theorem 3.2 on the unit interval. Let $U$ be a Kurtz test. Then there exists a computable sequence $\left\{\left(p_{n}, q_{n}\right)\right\}$ of pairwise disjoint base sets such that $U \backslash \bigcup_{n}\left(p_{n}, q_{n}\right)$ is a set of rationals where $p_{n}, q_{n} \in \mathbb{Q} \cap \mathbb{I}$ and $n \geq 1$. Let $U_{n}=\bigcup_{k=1}^{n}\left(p_{k}, q_{k}\right)$ and $U_{n} \uparrow U_{\infty}$. We can further assume that there exists a computable sequence $\left\{a_{m}\right\}$ of natural numbers such that $\mu\left(U_{a_{m}}\right)=1-2^{-m}$ and $a_{0}=0$.

Let $f: \mathbb{I} \rightarrow \overline{\mathbb{R}}$ be such that

$$
f(x)= \begin{cases}m-1+g_{\left\langle p_{n}, q_{n}\right\rangle}(x) & \text { if } x \in\left(p_{n}, q_{n}\right) \text { and } a_{m-1}<n \leq a_{m}, \\ \infty & \text { otherwise. }\end{cases}
$$

If $x \notin U$ then $f(x)=\infty$.

We prove $f \in \mathcal{K}_{\text {comp. }}$. The function $f$ is non-negative.

We prove that $f$ is extended computable. By Proposition 2.3 it suffices to show that $f^{-1}([0, q)), f^{-1}((p, q))$ and $f^{-1}((p, \infty])$ are uniformly c.e. open. Note that $\{x: f(x)<\infty\}=U_{\infty}$ and $f$ is computable on $U_{\infty}$. Hence $f^{-1}([0, q))$ and $f^{-1}((p, q))$ are c.e. open. To prove that $f^{-1}((p, \infty])$ is c.e. open, we show that $f^{-1}([0, p])$ is co-c.e. closed. Note that $f(\mathbb{I})=[0, \infty]$. Then

$$
\begin{aligned}
f^{-1}([0, p]) & =\{x: f(x) \leq p\} \\
& =\bigcup_{n}\left\{x \in\left(p_{n}, q_{n}\right): f(x) \leq q\right\} .
\end{aligned}
$$

Note that $\left\{x \in\left(p_{n}, q_{n}\right): f(x) \leq q\right\}$ is co-c.e. closed. Let $N$ be the minimum natural number such that $N>a_{m-1}$ and $m-1>q$. Then $f(x) \geq m-1>q$ for all $x \in\left(p_{n}, q_{n}\right)$ and $n \geq N$. Hence $f^{-1}([0, p])$ is the finite union of co-c.e. closed sets and it is co-c.e. closed.

Since $m$ depends on $n$, we write $m_{n}$. The integral $\mu(f)$ is

$$
\sum_{n=1}^{\infty} \int_{\left(p_{n}, q_{n}\right)}\left(m_{n}-1+g_{\left\langle p_{n}, q_{n}\right\rangle}\right) d \mu=\sum_{n=1}^{\infty}\left(q_{n}-p_{n}\right)\left(m_{n}-1\right)+\sum_{n=1}^{\infty}\left(q_{n}-p_{n}\right) G .
$$

The second term in the right-hand side is equal to $G$ because $\mu(U)=\mu\left(U_{\infty}\right)=1$ and $\mu\left(\bigcup_{n=1}^{\infty}\left(p_{n}, q_{n}\right)\right)=1$. Note that

$$
\sum_{n}\left\{\left(q_{n}-p_{n}\right): m_{n}=k\right\}=2^{-k}
$$


Thus

$$
\sum_{n=1}^{\infty}\left(q_{n}-p_{n}\right)\left(m_{n}-1\right)=\sum_{k=1}^{\infty}(k-1) 2^{-k} .
$$

Hence $\mu(f)$ is computable.

\subsection{Proof for computable metric spaces}

In this subsection we prove Theorem 3.2 for a computable metric space with a computable measure on it. The idea is similar to the case of the unit interval.

Definition 3.4 (inner approximation). A sequence $\left\{V_{n}\right\}$ of subsets of $X$ is an inner approximation for a set $U \subseteq X$ if

(i) $V_{n}$ is uniformly computable elements in $\mathcal{I}$,

(ii) $\left\{V_{n}\right\}$ is pairwise disjoint,

(iii) $\bigcup_{n} V_{n} \subseteq U$,

(iv) $\mu\left(U \backslash \bigcup_{n} V_{n}\right)=0$.

Lemma 3.5. From a c.e. open set $U$, one can uniformly construct an inner approximation $\left\{V_{n}\right\}$.

Proof. Since $U$ is a c.e. open set, there exists a computable sequence $\left\{B\left(v_{n}, s_{n}\right)\right\}$ of basic sets such that $U=\bigcup_{n} B\left(v_{n}, s_{n}\right)$. Let

$$
U_{n}=\bigcup_{k=1}^{n} B\left(v_{k}, s_{k}\right) \text { and } D_{n}=U_{n} \backslash U_{n-1}
$$

where $U_{0}=\emptyset$. Let

$$
V_{n}=U_{n} \cap \bigcap_{k=1}^{n-1} \bar{B}^{c}\left(v_{k}, s_{k}\right) .
$$

Then $V_{n} \in \mathcal{I}$ for each $n$. Then

$$
x \in \bigcap_{k=1}^{n-1} \bar{B}^{c}\left(v_{k}, s_{k}\right) \Rightarrow x \notin U_{n-1} .
$$

Hence $V_{n} \subseteq D_{n}$ and $\bigcup_{n} V_{n} \subseteq U$. Since $\left\{D_{n}\right\}$ is pairwise disjoint, $\left\{V_{n}\right\}$ is pairwise disjoint. Furthermore

$$
\mu\left(U \backslash \bigcup_{n} V_{n}\right)=\sum_{n=1}^{\infty} \mu\left(D_{n} \backslash V_{n}\right)=0 .
$$


Note that the union $\bigcup_{n} V_{n}$ of an inner approximation $\left\{V_{n}\right\}$ for a Kurtz test is a Kurtz test. We will finally construct a function $f \in \mathcal{K}_{\text {comp }}\left(\bigcup_{n} V_{n}\right)$. In the following we construct a partial computable function in $\mathcal{K}_{\text {comp }}\left(V_{n}\right)$ and combine them later.

On the unit interval we constructed a function $g_{\langle p, q\rangle}$ for each basic set $(p, q)$. Similarly we construct a function $f$ for each basic set $B(u, r)$.

Lemma 3.6. Let $\left\{x_{n}\right\}$ be a sequence of uniformly computable positive reals. If there exists a uniformly computable sequence $\left\{y_{n}\right\}$ such that $x_{n} \leq y_{n}$ for all $n$ and $\sum_{n} y_{n}$ is computable, then $\sum_{n} x_{n}$ is also computable.

Proof. Let $\left\{a_{n}\right\}$ be a computable sequence such that $\sum_{k=a_{n}+1}^{\infty} y_{k}<2^{-n}$. Since $x_{n} \leq y_{n}$ for all $n, \sum_{k=a_{n}+1}^{\infty} x_{n}<2^{-n}$. It follows that

$$
\left|\sum_{k=1}^{\infty} x_{k}-\sum_{k=1}^{a_{n}} x_{k}\right|<2^{-n}
$$

Since $\sum_{k=1}^{a_{n}} x_{k}$ is computable, $\sum_{n} x_{n}$ is also computable.

Lemma 3.7. One can uniformly construct a function $f \in \mathcal{K}_{\text {comp }}(D)$ from a basic set $D=B(u, r)=B_{\langle i, j\rangle}$.

Proof. Let $V=\mu(D)$. One can construct a computable sequence $\left\{s_{n}\right\}_{n} \subseteq\left\{r_{n}\right\}_{n}$ of reals such that

(i) $s_{0}=0$,

(ii) $s_{n-1}<s_{n}<r$,

(iii) $\mu\left(B\left(u, s_{n}\right)\right) \geq\left(1-2^{-n}\right) V$ for all $n$ and

(iv) $s_{n} \rightarrow r$ as $n \rightarrow \infty$.

Let $D_{n}=B\left(u, s_{n}\right) \backslash B\left(u, s_{n-1}\right)$ for all $n \geq 1$. Then

$$
\mu\left(\bigcup_{k=1}^{n} D_{k}\right)=\mu\left(B\left(u, s_{n}\right)\right) \geq\left(1-2^{-n}\right) \mu(D) .
$$

Then

$$
V=\mu\left(\bigcup_{k=1}^{n-1} D_{k}\right)+\mu\left(D_{n}\right)+\mu\left(\bigcup_{k=n+1}^{\infty} D_{k}\right) \geq \mu\left(\bigcup_{k=1}^{n-1} D_{k}\right)+\mu\left(D_{n}\right) .
$$

Hence $\mu\left(D_{n}\right) \leq V-\left(1-2^{-n+1}\right) V=2^{-n+1} V$.

Define $g: \mathbb{R}^{+} \rightarrow \overline{\mathbb{R}}$ by

$$
g(x)= \begin{cases}n-1+\frac{x-s_{n-1}}{s_{n}-s_{n-1}} & \text { if } s_{n-1} \leq x<s_{n} \text { for } n \geq 1 \\ \infty & \text { if } x \geq r .\end{cases}
$$


Then $g$ is non-negative. Note that $g$ is continuous at $x=s_{n}$ for each $n$. Then $g$ is continuous and a polygonal function. Hence $g$ is extended computable. Note that $g(x)<\infty$ iff $x<r$. Also note that $g$ is increasing.

Define $f: X \rightarrow \overline{\mathbb{R}}$ by

$$
f(x)=g(d(u, x)) .
$$

Then $f$ is non-negative and extended computable. Note that

$$
f(x)<\infty \Longleftrightarrow g(d(u, x))<\infty \Longleftrightarrow d(u, x)<r \Longleftrightarrow x \in D .
$$

We claim that $\int_{D} f d \mu$ is computable. Note that $B\left(u, s_{n}\right)$ has a computable measure and $f$ is a bounded computable function on $B\left(u, s_{n}\right)$ for each $n$. By Proposition 2.6 $\int_{B\left(u, s_{n}\right)} f d \mu$ is computable uniformly in $n$. Then

$$
\begin{aligned}
\int_{B\left(u, s_{n}\right)} f d \mu & =\int_{B\left(u, s_{n-1}\right)} f d \mu+\int_{D_{n}} f d \mu \\
& =\int_{B\left(u, s_{n-1}\right)} f d \mu+\int_{B\left(u, s_{n}\right) \cap \bar{B}^{c}\left(u, s_{n-1}\right)} f d \mu .
\end{aligned}
$$

The two integrations are lower semi-computable by Proposition 2.5. Since the sum of them is computable, they are computable. Hence $\int_{D_{n}} f d \mu$ is uniformly computable.

Furthermore

$$
\int_{D_{n}} f d \mu \leq n \mu\left(D_{n}\right) \leq n 2^{-n+1} V
$$

and $\sum_{n=1}^{\infty} n 2^{-n+1} V$ is computable. By Lemma 3.6

$$
\mu(f)=\sum_{n=1}^{\infty} \int_{D_{n}} f d \mu
$$

is also computable.

Similarly such a function can be constructed for a co-basic set.

Lemma 3.8. One can uniformly construct a function $f \in \mathcal{K}_{\text {comp }}(E)$ from a co-basic set $E=\bar{B}^{c}(u, r)=\bar{B}_{\langle i, j\rangle}^{c}$.

Proof. We can assume that $d(x, y)<1$ for all $x, y \in X$. Let $V=\mu(D)=$ $\mu(B(u, r))$. One can construct a computable sequence $\left\{t_{n}\right\}_{n} \subseteq\left\{r_{n}\right\}_{n}$ of reals such that

(i) $t_{0}=1$,

(ii) $r<t_{n}<t_{n-1}$,

(iii) $\mu\left(\bar{B}^{c}\left(u, t_{n}\right)\right) \geq\left(1-2^{-n}\right)(1-V)$ for all $n$ and

(iv) $t_{n} \rightarrow r$ as $n \rightarrow \infty$. 
Define $g: \mathbb{R}^{+} \rightarrow \overline{\mathbb{R}}$ by

$$
g(x)= \begin{cases}n-1+\frac{t_{n-1}-x}{t_{n-1}-t_{n}} & \text { if } t_{n} \leq x<t_{n-1} \text { for } n \geq 1 \\ \infty & \text { if } x \leq r .\end{cases}
$$

Define $f: X \rightarrow \overline{\mathbb{R}}$ by $f(x)=g(d(u, x))$.

By an argument similar to the proof of Lemma 3.7, $f$ is non-negative and extended computable, $f(x)<\infty \Longleftrightarrow x \in E, \int_{E} f d \mu$ is computable.

Lemma 3.9. One can uniformly construct a function $f \in \mathcal{K}_{\text {comp }}(U)$ from an open set $U \in \mathcal{I}$.

Proof. Since $U \in \mathcal{I}, U$ can be written as

$$
U=\bigcap_{n \leq N} B\left(v_{n}, s_{n}\right) \cap \bigcap_{m \leq M} \bar{B}^{c}\left(w_{m}, t_{m}\right) .
$$

Let $f_{n}^{i}$ be a function for $B\left(v_{n}, s_{n}\right)$ as in Lemma 3.7 and $f_{m}^{o}$ be a function for $\bar{B}^{c}\left(w_{m}, t_{m}\right)$ as in Lemma 3.8 uniformly in $n$ and $m$. Define $f: X \rightarrow \overline{\mathbb{R}}$ by

$$
f(x)=\sum_{n \leq N} f_{n}^{i}(x)+\sum_{m \leq M} f_{m}^{o}(x) .
$$

Then $f$ is non-negative and extended computable.

We claim that $f(x)<\infty \Longleftrightarrow x \in U$. This is because

$$
\begin{aligned}
f(x)<\infty & \Longleftrightarrow f_{n}^{i}(x)<\infty \text { for all } n \leq N \text { and } f_{m}^{i}(x)<\infty \text { for all } m \leq M \\
& \Longleftrightarrow x \in \bigcap_{n \leq N} B\left(v_{n}, s_{n}\right) \cap \bigcap_{m \leq M} \bar{B}^{c}\left(w_{m}, t_{m}\right) \\
& \Longleftrightarrow x \in U .
\end{aligned}
$$

We show that $\int_{U} f_{n}^{i} d \mu$ and $\int_{U} f_{m}^{o} d \mu$ are computable uniformly. Note that

$$
\int_{U} f d \mu=\sum_{n \leq N} \int_{U} f_{n}^{i} d \mu+\sum_{m \leq M} \int_{U} f_{m}^{o} d \mu .
$$

Then $\int_{U} f d \mu$ is computable. Hence $f \in \mathcal{K}_{\text {comp }}(U)$.

As an example, we show that $\int_{U} f_{1}^{i} d \mu$ is computable. We divide $B\left(v_{1}, s_{1}\right)$ into $2^{N+M-1}$ pairwise disjoints sets $\left\{V_{k}\right\}$. For $k=1, \ldots, 2^{N+M-1}$, let

$$
V_{k}=B\left(v_{1}, s_{1}\right) \cap \bigcap_{1<n \leq N} Y_{n} \cap \bigcap_{m \leq M} Z_{m}
$$

where

$$
\begin{gathered}
Y_{n}=B\left(v_{n}, s_{n}\right) \text { or } \bar{B}^{c}\left(v_{n}, s_{n}\right), \\
Z_{m}=B\left(w_{m}, t_{m}\right) \text { or } \bar{B}^{c}\left(w_{m}, t_{m}\right) .
\end{gathered}
$$


Note that there exists $k$ such that $V_{k}=U$. Then $\bigcup_{k} V_{k} \subseteq B\left(v_{1}, s_{1}\right)$ and $\mu\left(B\left(v_{1}, s_{1}\right)\right)=\mu\left(\bigcup_{k} V_{k}\right)$. For each $k$, the integration $\int_{V_{k}} f_{1}^{i} d \mu$ is lower semicomputable uniformly. The sum of them is equal to $\int_{B\left(v_{1}, s_{1}\right)} f_{1}^{i} d \mu$ and is computable by the definition of $f_{1}^{i}$. Hence $\int_{V_{k}} f_{1}^{i} d \mu$ is uniformly computable. In particular $\int_{U} f_{1}^{i} d \mu$ is computable.

Proof of (iv) $\Rightarrow$ (i) of Theorem 3.2. Let $\left\{V_{n}\right\}$ be an inner approximation for a Kurtz test $U$. Let $U_{n}=\bigcup_{k=1}^{n} V_{k}$. Recall that $\mu\left(V_{n}\right)$ is uniformly computable. Hence one can construct a strictly increasing computable sequence $\left\{a_{m}\right\}$ of natural numbers such that $\mu\left(U_{a_{m}}\right)>1-2^{-m}$ and $a_{0}=0$.

Let $f_{n} \in \mathcal{K}_{\text {comp }}\left(V_{n}\right)$ be uniform by Lemma 3.9. We can further assume that $\int_{V_{n}} f_{n} d \mu=\mu\left(V_{n}\right)$. Define $f: X \rightarrow \overline{\mathbb{R}}$ by

$$
f(x)= \begin{cases}m-1+f_{n}(x) & \text { if } x \in V_{n} \text { and } a_{m-1}<n \leq a_{m} \\ \infty & \text { if } x \notin \bigcup_{n} V_{n}\end{cases}
$$

Then $f$ is non-negative because $f_{n}$ is non-negative. If $x \notin U$, then $f(x)=\infty$.

We claim that $f$ is extended computable. Note that $f$ is computable on $V_{n}$ for each $n$. Hence it suffices to show that $f^{-1}((q, \infty])$ is uniformly c.e. open for each $q \in \mathbb{Q}$. For each $n$ and $m$ such that $a_{m-1}<n \leq a_{m}$,

$$
V_{n}^{c} \subseteq\left\{x \in X: m-1+f_{n}(x)>q\right\} .
$$

Then

$$
\left\{x \in X: m-1+f_{n}(x) \leq q\right\} \subseteq V_{n} .
$$

It follows that $\left\{x \in V_{n}: f(x) \leq q\right\}$ is co-c.e. closed. Note that the set $\left\{x \in V_{n}: f(x) \leq q\right\}$ is empty for all but finitely many $n$. Hence

$$
f^{-1}([0, q])=\bigcup_{n}\left\{x \in V_{n}: f(x) \leq q\right\} .
$$

is co-c.e. closed. Since $f$ is surjective, $f^{-1}((q, \infty])$ is uniformly c.e. open.

We claim that $\mu(f)$ is computable. Note that

$$
\int_{V_{n}} f d \mu=(m-1) \mu\left(V_{n}\right)+\int_{V_{n}} f_{n} d \mu=(m-1) \mu\left(V_{n}\right)+\mu\left(V_{n}\right)=m \cdot \mu\left(V_{n}\right)
$$

is computable. Then

$$
\sum_{a_{m-1}<n \leq a_{m}} \int_{V_{n}} f d \mu=m\left(\mu\left(U_{a_{m}}\right)-\mu\left(U_{a_{m-1}}\right)\right) \leq m\left(1-\left(1-2^{-m+1}\right)\right)=m \cdot 2^{-m+1} .
$$

By Lemma 3.6

$$
\mu(f)=\sum_{m=1}^{\infty} \sum_{a_{m-1}<n \leq a_{m}} \int_{V_{n}} f_{n} d \mu
$$

is computable. 


\subsection{Characterization by a differentiation theorem}

We gave some characterizations of Kurtz randomness by integral tests in Theorem 3.2. This theorem gives a characterization of Kurtz randomness by a differentiation theorem.

Definition 3.10 (Lebesgue point). Let $(X, d)$ be a metric space and $f: \subseteq X \rightarrow$ $\overline{\mathbb{R}}$ be a function. A point $x \in X$ is a Lebesgue point for $f$ if

$$
\lim _{r \rightarrow 0} \frac{1}{\mu B(x, r)} \int_{B(x, r)} f d \mu=f(x),
$$

and the both sides are defined and are finite. We write $L(f)$ to mean the set of Lebesgue points for $f$.

The differentiation theorem says that $\mu(L(f))=1$ if $X=\mathbb{R}^{n}$. Note that each point $x \in X$ is a Lebesgue point for every continuous function $f: X \rightarrow \mathbb{R}$. Then $x \in X$ is a Lebesgue point for a non-negative extended computable function $f: X \rightarrow \overline{\mathbb{R}}$ iff $f(x)<\infty$. Hence finiteness in Theorem 3.2 can be replaced with being a Lebesgue point.

In particular we have the following on the unit interval.

Corollary 3.11. Let $\mathbf{I}$ be the unit interval in Example 2.2. A point $z \in[0,1]$ is Kurtz random iff each computable function $F:[0,1] \rightarrow \mathbb{R}$ whose derivative is non-negative and extended computable is differentiable at $z$.

Proof. Suppose that $z$ is not Kurtz random. Then there exists a non-negative extended computable function $f: X \rightarrow \overline{\mathbb{R}}$ such that $\mu(f)$ is a computable real and $f(z)=\infty$. Let

$$
F(x)=\int_{0}^{x} f(t) d t .
$$

Then $F$ is a computable function and the derivative of $F$ is $f$. Furthermore $F$ is not differentiable at $z$.

Suppose that $F$ is not differentiable at $z$ for a computable function $F$ whose derivative is extended computable. Let $f$ be the derivative of $F$. Then $f$ is nonnegative and extended computable. Furthermore $\mu(f)=F(1)$ is computable. Since $F$ is not differentiable at $z, f(z)=\infty$. Then $z$ is not Kurtz random.

\section{Removing non-negativeness}

\subsection{Some notations}

We define some other symbols used in the following. Recall that $\mathcal{I}$ is the set of all finite intersections of basic sets and co-basic sets. Let $\mathcal{U}$ be the set of all finite unions of elements in $\mathcal{I}$. For a set $E \in \mathcal{U}$, we define $C(E)$ inductively as follows.

(i) If $E$ is a basic set $B(u, r)$, then $C(E)=\bar{B}^{c}(u, r)$. 
(ii) If $E$ is a co-basic set $\bar{B}^{c}(u, r)$, then $C(E)=B(u, r)$.

(iii) If $E=E_{1} \cap \cdots \cap E_{n} \in \mathcal{I}$ where $E_{i}$ is a basic set or a co-basic set, then $C(E)=C\left(E_{1}\right) \cup \cdots \cup C\left(E_{n}\right) \in \mathcal{U}$.

(iv) If $E=E_{1} \cup \cdots \cup E_{n}$ where $E_{i} \in \mathcal{I}$, then $C(E)=C\left(E_{1}\right) \cap \cdots \cap C\left(E_{n}\right) \in \mathcal{U}$.

Then the following is easy to prove by induction.

Lemma 4.1. For each $E \in \mathcal{U}, E \cap C(E)=\emptyset, \mu(E \cup C(E))=1$ and $\mu(E)$ is uniformly computable.

Let $\mathcal{D}$ be the set of two functions in $\mathcal{K}$, that is, $\mathcal{D}=\{f-g: f, g \in \mathcal{K}\}$. Here $\operatorname{dom}(f-g)=\{x: f(x)<\infty$ and $g(x)<\infty\}$. Similarly $\mathcal{D}_{\text {fin }}$ and $\mathcal{D}_{\text {comp }}$ are defined. The symbols $\mathcal{A}, \mathcal{A}_{\text {fin }}$ and $\mathcal{A}_{\text {comp }}$ are defined just after Definition 4.3 .

Definition 4.2 (Kurtz equivalence). Two functions $f, g: X \rightarrow \overline{\mathbb{R}}$ are Kurtz equivalent on $E \subseteq X$ if $f(x)=g(x)$ for each Kurtz random point $x \in E$. If $E=X$, then we omit "on $X$ ".

\subsection{Almost everywhere computability}

We gave some characterizations of Kurtz randomness by the differentiation theorem. In the following, we will remove the non-negativeness and values $\infty$.

A naive way to do this is to take the difference of two functions. Recall that $\mathcal{K}$ is the set of non-negative extended computable functions $f: X \rightarrow \overline{\mathbb{R}}$ such that $f(x)<\infty$ almost everywhere. Then the following are equivalent.

(i) $z$ is Kurtz random.

(ii) $z$ is a Lebesgue point for each $h \in \mathcal{D}$.

(iii) $z$ is a Lebesgue point for each $h \in \mathcal{D}_{\text {fin }}$.

(iv) $z$ is a Lebesgue point for each $h \in \mathcal{D}_{\text {comp }}$.

The question is whether $\mathcal{D}, \mathcal{D}_{\text {fin }}$ and $\mathcal{D}_{\text {comp }}$ have other simple characterizations. We give a partial answer.

Definition 4.3. We say that a function $f: \subseteq X \rightarrow \mathbb{R}$ is almost everywhere computable (or a.e. computable) if it is computable and is defined almost everywhere.

Note that a function $f$ is a.e. computable if and only if the two partial functions $f$ and $-f$ are $\left(\delta, \rho_{<}\right)$-computable and they are defined almost everywhere, which is equivalent to that there are a lower semi-computable function $\underline{f}: X \rightarrow \overline{\mathbb{R}}$ and an upper semi-computable function $\bar{f}: X \rightarrow \overline{\mathbb{R}}$ such that

(i) $\underline{f}(x) \leq \bar{f}(x)$ for all $x \in X$, 
(ii) $\operatorname{dom}(f)=\{x: \underline{f}(x)=\bar{f}(x)\}$,

(iii) $\mu(\operatorname{dom}(f))=1$,

(iv) $f(x)=\underline{f}(x)$ for $x \in \operatorname{dom}(f)$.

Let $\mathcal{A}$ be the set of all a.e. computable functions. Let $\mathcal{A}_{\text {fin }}$ and $\mathcal{A}_{\text {comp }}$ be the subset of $\mathcal{A}$ restricted to the functions $f$ such that $\mu(|f|)<\infty$ and $\mu(|f|)$ is computable respectively. Clearly

$$
\mathcal{A}_{\text {comp }} \subseteq \mathcal{A}_{\text {fin }} \subseteq \mathcal{A} .
$$

Note that

$$
\mathcal{D}_{\text {comp }} \subseteq \mathcal{A}_{\text {comp }}, \mathcal{D}_{\text {fin }} \subseteq \mathcal{A}_{\text {fin }}, \mathcal{D} \subseteq \mathcal{A} .
$$

The converses of the latter two hold in a weak form.

Proposition 4.4. Let $h$ be an a.e. computable function. For each $n \in \mathbb{N}$, there exists a function $g \in \mathcal{D}$ such that $|h(x)-g(x)| \leq 2^{-n}$ on Kurtz random points. If $h \in \mathcal{A}_{\text {fin }}$, then $g$ can satisfy $g \in \mathcal{D}_{\text {fin }}$.

The goal of this subsection is to prove this proposition. We prepare definitions and lemmas. Let $\overline{\mathbb{Q}}=\mathbb{Q} \cup\{ \pm \infty\}$.

Definition 4.5. A function $h: X \rightarrow \overline{\mathbb{R}}$ is computably simple if

(i) $\operatorname{rng}(h)$ is a finite subset of $\overline{\mathbb{Q}}$,

(ii) $h^{-1}((q, \infty])$ is a finite union of basic sets uniformly in $q \in \mathbb{Q}$.

Lemma 4.6. A function $h: X \rightarrow \overline{\mathbb{R}}$ is lower semi-computable iff there exist a sequence $\left\{h_{s}\right\}$ of uniformly computably simple functions such that $h_{s}(x) \leq$ $h_{s+1}(x)$ and $\lim _{s \rightarrow \infty} h_{s}(x)=h(x)$ for all $x$.

We say $h_{s}$ is a computable approximation of $h$.

Proof. Let $\left\{q_{i}\right\}$ be a computable enumeration of elements in $\overline{\mathbb{Q}}$ such that $q_{0}=$ $-\infty$. Since $h$ is lower semi-computable, $h^{-1}\left(\left(q_{i},+\infty\right]\right)$ is uniformly c.e. Hence there exists an infinite sequence $\left\{U_{n}^{i}\right\}$ of basic sets such that $h^{-1}\left(\left(q_{i},+\infty\right]\right)=$ $\bigcup_{n} U_{n}^{i}$. Here we allow $U_{n}^{i}=U_{m}^{i}$ for $n \neq m$. Let

$$
h_{s}(x)=\max \left\{q_{i}: x \in U_{n}^{i} \text { for } i<s \wedge n<s\right\} .
$$

If the set is empty, $h_{s}(x)=-\infty$.

We claim that $h_{s}$ is computably simple. The range $\operatorname{rng}\left(h_{s}\right) \subseteq\left\{q_{i}: i<\right.$ $s\} \subseteq \overline{\mathbb{Q}}$. For each $q \in \mathbb{Q}$,

$$
h_{s}^{-1}((q, \infty])=\bigcup\left\{U_{n}^{i}: q<q_{i} \wedge i<s \wedge n<s\right\} .
$$

Hence $h_{s}^{-1}((q, \infty])$ is a finite union of basic sets. It follows that $h_{s}$ is computably simple.

By the definition of $h_{s}$, it is immediate that $h_{s}(x) \leq h_{s+1}(x)$ for all $x$.

We claim that $\lim _{s \rightarrow \infty} h_{s}(x)=h(x)$. Let $x \in X$ and $q_{i} \in \mathbb{Q}$ be such that $h(x)>q_{i}$. Then $x \in h^{-1}\left(\left(q_{i}, \infty\right]\right)$. It follows that $x \in U_{n}^{i}$ for some $n$. Hence $h_{s}(x) \geq q_{i}$ for $s \geq n$. Therefore $\lim _{s \rightarrow \infty} h_{s}(x)=h(x)$. 
Definition 4.7. A function $h: X \rightarrow \overline{\mathbb{R}}$ is computably supersimple if

(i) $\operatorname{rng}(h)$ is a finite subset of $\overline{\mathbb{Q}}$,

(ii) $\{x: h(x)=q\} \in \mathcal{U}$ uniformly in $q \in \mathbb{Q}$.

Recall that two functions $f, g: X \rightarrow \overline{\mathbb{R}}$ are Kurtz equivalent if $f(x)=g(x)$ for each Kurtz random point $x \in X$.

Lemma 4.8. Each computably simple function $h$ is Kurtz equivalent to a computably supersimple function $g$ on $\operatorname{dom}(h)$.

Proof. Let $\left\{q_{i}\right\}$ be a computable enumeration of elements in $\overline{\mathbb{Q}}$ such that $q_{0}=$ $-\infty$. Let $s \in \mathbb{N}$ be such that

$$
\operatorname{rng}(h) \subseteq\left\{q_{i}: i<s\right\} .
$$

Let $p_{i}$ be the $(i+1)$-st element in $\left\{q_{i}: i<s\right\}$ in increasing order. Then $p_{0}=-\infty$.

For $i$ such that $0<i<s$ let

$$
V_{i}=\left\{x: h(x)>p_{i-1}\right\} \cap C\left(\left\{x: h(x)>p_{i}\right\}\right) \in \mathcal{U} .
$$

Let

$$
g(x)= \begin{cases}h(x) & \text { if } x \in V_{i} \text { for some } i \\ -\infty & \text { otherwise }\end{cases}
$$

Then $\operatorname{rng}(g) \subseteq \operatorname{rng}(h)$ and

$$
\left\{x: g(x)=p_{i}\right\}=V_{i} \in \mathcal{U}
$$

for $0<i<s$. Hence $g$ is a computably supersimple function.

Note that

$$
\left\{x: h(x)=p_{i}\right\}=\left\{x: h(x)>p_{i-1}\right\} \backslash\left\{x: h(x)>p_{i}\right\} .
$$

Since $\mu\left(C\left(\left\{x: h(x)>p_{i}\right\}\right) \cup\left\{x: h(x)>p_{i}\right\}\right)=1$ by Lemma 4.1,

$$
\mu\left(\left\{x: h(x)=p_{i}\right\}\right)=\mu\left(V_{i}\right) .
$$

Let $V_{0}=C(\{x: h(x)>-\infty\})$. Then

$$
\mu(\{x: h(x)=-\infty\})=\mu\left(V_{0}\right) .
$$

Since $\mu\left(\bigcup_{i<s}\left\{x: h(x)=p_{i}\right\}\right)=\mu(X)=1, \mu\left(\bigcup_{n} V_{n}\right)=1$. Hence $\bigcup_{n} V_{n}$ is a Kurtz test.

Let $x \in \operatorname{dom}(h)$ be a Kurtz random point. It follows that $x \in V_{i}$ for some $i$. Hence $g(x)=h(x)$. 
Lemma 4.9. Let $\left\{q_{i}\right\}$ be a computable sequence of rational numbers and $\left\{V_{i}\right\}$ be an inner approximation for $X$. Define a function $h: \subseteq X \rightarrow \mathbb{R}$ as

$$
h=\sum_{i} q_{i} \mathbf{1}_{V_{i}} .
$$

Then $h \in \mathcal{D}$. If $\sum_{i}\left|q_{i}\right| \mu\left(V_{i}\right)<\infty$, then $h \in \mathcal{D}_{\text {fin }}$.

Proof. Let $f$ be the function constructed from $\left\{V_{i}\right\}$ in the proof of the "if" direction of Theorem 3.2. Define $g_{1}, g_{2}: X \rightarrow \overline{\mathbb{R}}$ by

$$
\begin{gathered}
g_{1}(x)= \begin{cases}q_{i}+f(x) & \text { if } x \in V_{i} \text { and } q_{i} \geq 0 \\
f(x) & \text { if } x \in V_{i} \text { and } q_{i}<0 \\
\infty & \text { otherwise. }\end{cases} \\
g_{2}(x)= \begin{cases}f(x) & \text { if } x \in V_{i} \text { and } q_{i} \geq 0 \\
-q_{i}+f(x) & \text { if } x \in V_{i} \text { and } q_{i}<0 \\
\infty & \text { otherwise. }\end{cases}
\end{gathered}
$$

Then $g_{1}$ and $g_{2}$ are non-negative and extended computable and $g_{j}(x)<\infty \Longleftrightarrow$ $x \in \bigcup_{i} V_{i}$ for $j=1,2$. Then $g_{1}, g_{2} \in \mathcal{K}$ and $g_{1}-g_{2} \in \mathcal{D}$. Also note that $h(x)=g_{1}(x)-g_{2}(x)$ for $x \in \bigcup_{i} V_{i}$.

Suppose that $\sum_{i}\left|q_{i}\right| \mu\left(V_{i}\right)<\infty$. Then

$$
\mu\left(g_{j}\right) \leq \sum_{i}\left|q_{i}\right| \mu\left(V_{i}\right)+\mu(f)<\infty .
$$

Then $g_{1}, g_{2} \in \mathcal{K}_{\text {fin }}$ and $h \in \mathcal{D}_{\text {fin }}$.

Proof of Proposition 4.4. Let $\bar{h}$ and $\underline{h}$ be functions for $h$ as in Definition 4.3. Note that $-\bar{h}$ and $\underline{h}$ are lower semi-computable. By Lemma 4.6 let $-\bar{h}_{s}$ and $\underline{h}_{s}$ be computable approximations of $-\bar{h}$ and $\underline{h}$ respectively.

Let

$$
U_{s}=\left\{x: \bar{h}_{s}(x)-\underline{h}_{s}(x)<2^{-n}\right\} .
$$

Then

$$
U_{s}=\bigcup_{q \in \mathbb{Q}}\left(\left\{x: \bar{h}_{s}(x)<q\right\} \cap\left\{x: \underline{h}_{s}(x)>q-2^{-n}\right\}\right) \in \mathcal{U} .
$$

Let $V_{s}=U_{s} \cap C\left(U_{s-1}\right) \in \mathcal{U}$. Then $\left\{V_{s}\right\}$ is pairwise disjoint and $\mu\left(\bigcup_{n} V_{s}\right)=1$.

Let $\underline{h}_{s}^{\prime}$ be a computably supersimple function that is Kurtz equivalent to $\underline{h}_{s}$ for each $s$ by Lemma 4.8. Let

$$
g(x)=\underline{h}_{s}^{\prime}(x) \text { if } x \in V_{s} .
$$

Then

$$
|h(x)-g(x)|=h(x)-\underline{h}_{s}^{\prime}(x) \leq \bar{h}_{s}(x)-\underline{h}_{s}(x)<2^{-n} .
$$

if $x \in V_{s}$ and $x$ is Kurtz random. Hence $|h(x)-g(x)|<2^{-n}$ on Kurtz random points. 
From an a.e. computable function $h$, construct a Let

$$
E_{i}^{s}=\left\{x: \underline{h}_{s}^{\prime}(x)=q_{i}\right\} \in \mathcal{U}
$$

where $\left\{q_{i}\right\}$ is a computable enumeration of elements in $\overline{\mathbb{Q}}$. Then

$$
\underline{h}_{s}=\sum_{q_{i} \in \overline{\mathbb{Q}}} q_{i} \mathbf{1}_{E_{i}^{s}} .
$$

Let

$$
g=\sum_{s} \sum_{q_{i} \in \mathbb{Q}} q_{i} \mathbf{1}_{E_{i}^{s} \cap V_{s}} .
$$

Note that $\underline{h}_{s}(x) \neq-\infty$ for $x \in V_{s}$.

Since $E_{i}^{s} \cap V_{s}$ is a c.e. open set, it has an inner approximation. Then there exists a sequence $W_{i}$ such that $\left\{W_{\langle i, s, m\rangle}\right\}_{m}$ is a inner approximation for $E_{i}^{s} \cap V_{s}$. Let $p_{\langle i, s, m\rangle}=q_{i}$. Then

$$
g=\sum_{j} p_{j} \mathbf{1}_{W_{j}} .
$$

Now the proposition follows from Lemma 4.9.

\subsection{The differentiation theorem}

Proposition 4.10. For a point $z \in X$, the following are equivalent.

(i) $z$ is Kurtz random.

(ii) $f(z)$ is defined for each a.e. computable function $f: \subseteq X \rightarrow \mathbb{R}$.

(iii) $z$ is a Lebesgue point of each a.e. computable function $f: \subseteq X \rightarrow \mathbb{R}$.

The equivalence between (i) and (ii) on the unit interval was given by Jason Rute (personal communication). Here we give another proof in the general setting of a computable metric space.

Proof. (iii) $\Rightarrow$ (ii) If $z$ is a Lebesgue point for a function $f \in \mathcal{A}$, then $f(z)$ is defined.

(ii) $\Rightarrow($ i) Suppose $z$ is not Kurtz random. Then there exists a function $f \in \mathcal{K}$ such that $f(z)=\infty$. Let $g=f-0$ where 0 is the constant function. Then $g \in \mathcal{D} \subseteq \mathcal{A}$ and $g(z)$ is not defined.

(i) $\Rightarrow$ (iii) Suppose $z$ is Kurtz random and $f$ be in $\mathcal{A}$. By the proof of Proposition 4.4 , for each $n$, there exists a computably supersimple function $g_{n}$ and an open 
set $V \in \mathcal{U}$ such that $g_{n}(x)=g_{n}(y)$ for $x, y \in V$ and $\left|f(x)-g_{n}(x)\right| \leq 2^{-n}$ for $x \in V$. Then, for each $r$ such that $B(z, r) \subseteq V$,

$$
\begin{aligned}
& \int_{B(z, r)}|f(x)-f(z)| d \mu \\
= & \int_{B(z, r)}\left(\left|f(x)-g_{n}(x)\right|+\left|g_{n}(x)-g_{n}(z)\right|+\left|g_{n}(z)-f(z)\right|\right) d \mu \\
\leq & 2^{-n+1} \mu B(z, r) .
\end{aligned}
$$

Since $n$ is arbitrary,

$$
\lim _{r \rightarrow 0} \frac{1}{\mu B(z, r)} \int_{B(z, r)}|f(x)-f(z)| d \mu \rightarrow 0 .
$$

Hence $z$ is a Lebesgue point.

\section{When two functions are Kurtz equivalent}

The following is a classical result. For a function $f: X \rightarrow \overline{\mathbb{R}}, \int|f| d \mu=0$ iff $f(x)=0$ almost everywhere. In this section we give an effectivization of this result.

The first idea is to restrict $f$ to be computable. Let $f: X \rightarrow \mathbb{R}$ be a computable function with $\int|f| d \mu=0$. Then $f$ is continuous and $f(x)=0$ for all $x \in X$. Hence computability is too strong to characterize Kurtz randomness.

As a simple corollary of Proposition 4.10, we have the following.

Theorem 5.1. A point $z$ is Kurtz random iff $f(z)=0$ for each a.e. computable function $f$ with $\int|f| d \mu=0$.

Proof. Let $z$ be a Kurtz random point and $f$ be a function in $\mathcal{A}$. By Proposition $4.10 z$ is a Lebesgue point. It follows that

$$
f(z)=\lim _{r \rightarrow 0} \frac{1}{\mu B(z, r)} \int_{B(z, r)} f(x) d \mu .
$$

Here the right-hand side is 0 because $\int|f| d \mu=0$.

Suppose $z$ is not Kurtz random. Then there exists a function $f \in \mathcal{K}$ such that $f(z)=\infty$. Let $g=f-f \in \mathcal{D} \subseteq \mathcal{A}$. Then $g(z)$ is not defined. Since $f(x)<\infty$ almost everywhere, $g(x)$ is defined almost everywhere. Hence $\int|g| d \mu=0$.

The theorem above is rewritten as follows.

Theorem 5.2. Let $f, g$ be a.e. computable functions. Then $f, g$ are Kurtz equivalent iff $\int|f-g| d \mu=0$.

Proof. Suppose that $f, g \in \mathcal{A}$ satisfy $\int|f-g| d \mu=0$. Since $f-g \in \mathcal{A},(f-$ $g)(x)=0$ for each Kurtz random point $x$ by Theorem 5.1. It follows that $f$ and $g$ are Kurtz equivalent.

Suppose that $f, g \in \mathcal{A}$ are Kurtz equivalent. Then $f(x)=g(x)$ almost everywhere. It follows that $\int|f-g| d \mu=0$. 


\section{Acknowledgement}

The author thanks Jason Rute and André Nies for the useful comments. The author also appreciates the anonymous referees for their careful reading and many comments. This work was partly supported by GCOE, Kyoto University and JSPS KAKENHI 23740072.

\section{References}

[1] A. S. Besicovitch. A general form of the covering principle and relative differentiation of additive functions. Proceedings of the Cambridge Philosophical Society, 41(2):103-110, 1945.

[2] V. Bosserhoff. Notions of probabilistic computability on represented spaces. Journal of Universal Computer Science, 14(6):956-995, 2008.

[3] V. Brattka. Computability over topological structures. In S. B. Cooper and S. S. Goncharov, editors, Computability and Models, pages 93-136. Kluwer Academic Publishers, New York, 2003.

[4] V. Brattka, P. Hertling, and K. Weihrauch. A tutorial on computable analysis. New Computational Paradigms, pages 425-491, 2008.

[5] V. Brattka, J. S. Miller, and A. Nies. Randomness and differentiability. Submitted.

[6] O. Demuth. The differentiability of constructive functions of weakly bounded variation on pseudo numbers. Comment. Math. Univ. Carolin., 16(3):583-599, 1975.

[7] R. Downey and D. R. Hirschfeldt. Algorithmic Randomness and Complexity. Springer, Berlin, 2010.

[8] C. Freer, B. Kjos-Hanssen, and A. Nies. Computable aspects of Lipshitz functions. In preparation.

[9] P. Gács. Uniform test of algorithmic randomness over a general space. Theoretical Computer Science, 341:91-137, 2005.

[10] P. Gács, M. Hoyrup, and C. Rojas. Randomness on Computable Probability Spaces - A Dynamical Point of View. Theory of Computing System, 48(3):465-485, 2011.

[11] S. Galatolo, M. Hoyrup, and C. Rojas. A constructive Borel-Cantelli lemma. Constructing orbits with required statistical properties. Theoretical Computer Science, 410(21-23):2207-2222, 2009.

[12] S. Galatolo, M. Hoyrup, and C. Rojas. Effective symbolic dynamics, random points, statistical behavior, complexity and entropy. Information and Computation, 208(1):23-41, 2010. 
[13] M. Hoyrup and C. Rojas. Computability of probability measures and Martin-Löf randomness over metric spaces. Information and Computation, 207(7):830-847, 2009.

[14] S. A. Kurtz. Randomness and Genericity in the Degrees of Unsolvability. $\mathrm{PhD}$ thesis, University of Illinois at Urbana-Champaign, 1981.

[15] H. Lebesgue. Leçons sur l'Intégration et la recherche des fonctions primitives. Gauthier-Villars, Paris, 1904.

[16] H. Lebesgue. Sur l'intégration des fonctions discontinues. Annales scientifiques de l'École Normale Supérieure, 27:361-450, 1910.

[17] M. Li and P. Vitányi. An introduction to Kolmogorov complexity and its applications. Graduate Texts in Computer Science. Springer-Verlag, New York, third edition edition, 2009.

[18] A. Nies. Computability and Randomness. Oxford University Press, USA, 2009.

[19] N. Pathak, C. Rojas, and S. G. Simpson. Schnorr randomness and the Lebesgue Differentiation Theorem. To appear in Proceedings of the American Mathematical Society.

[20] M. Schröder. Admissible Representations for Probability Measures. Mathematical Logic Quarterly, 53(4-5):431-445, 2007.

[21] J. Tiser. Differentiation Theorem for Gaussian Measures on Hilbert Space. Transactions of the American Mathematical society, 308(2):655-666, 1988.

[22] V. Vovk and V. Vyugin. On the empirical validity of the Bayesian method. Journal of the Royal Statistical Society. Series B (Methodological), 55(1):253-266, 1993.

[23] K. Weihrauch. Computable Analysis: an introduction. Springer, Berlin, 2000 .

[24] K. Weihrauch and T. Grubba. Elementary Computable Topology. Journal of Universal Computer Science, 15(6):1381-1422, 2009. 